\title{
IE CYLINDRE DES LANGAGES LINEAIRES N'EST PAS PRINCIPAL
}

L. BOASSON et M. NIVAT

La présente communication répond à une question posée par S. Greibach [i,4]: existe-t-il un langage linéaire dont tout langage linéaire soit image dans un homomorphisme inverse? La réponse est non ainsi qu'il est suggéré dans [4].

Rappelons qu'un langage linéajre est un langage algébrique (ou "context-free") engendrable par une granmaire a.lgébrique linéaire (i.e. dont les membres droits de règles contiennent au plus une lettre non-terminale). Par définition, une famille de langages $\mathscr{L}$ constitue un cylindre si elle est fermée par homomorphisme inverse et intersection par les langages rationnels (ou réguliers). Notons qu'un cylindre fermé par homomorphisme est un cône rationnel [1]. Un cylinare $\mathscr{Q}$ est principal s'il existe un langage I tel que le plus petit cylindre contenant I soit précisément $\mathscr{C}$. Le but de cette communication est d'établir le

Théorème: La famille Lin des langages linéaires constitue un cylindre qui n'est pas principal.

Notons que la famille Lin, qui constitue un cône rationnel, est principale en tant que cône et pas en tant que cylindre, alors qu'au contraire la famille des langages algébriques est principale non seulement en tant que cône rationnel, mais aussi en tant que cylindre [4]. La question reste ouverte de savoir si le cylindre des langages à compteur est principal ou non.

Nous donnons ci-dessous les principaux éléments nécessaires à l'établissement de la preuve du théorème annoncé.

A - Les langages $\hat{\mathrm{S}}_{\mathrm{n}}$ :

Sur I'alphabet $Z_{n}=Y_{n} \cup \bar{Y}_{n}$ où $Y_{n}=\left\{a_{i} \mid i=1, \ldots, n\right\}$ et $\bar{Y}_{n}=\left\{\bar{a}_{i} \mid i=1, \ldots, n\right\}$, on définit de façon classique le langage symétrique $S_{n}$ $s_{n}=\left\{a_{i_{1}} a_{i_{2}} \ldots a_{i_{p}} \bar{a}_{i_{p}} \ldots \bar{a}_{i_{2}} \bar{a}_{i_{1}} \mid p \geqslant 1 \quad a_{i j} \in Y_{n}\right\}$. Désignant par $R_{n}$ le langage rationnel $\left\{a_{i} \bar{a}_{i} \mid i=1, \ldots, n\right\}^{+}$, on construit le langage $S_{n}^{\prime \prime}$ à partir de $S_{n}$ en substituant dans ce dernier langage à chaque occurrence de $a \in Y$ le langage rationnel $a R_{n}$ et à chaque $\bar{a} \in \bar{Y}$ le langage rationnel ( $\bar{a} R_{n} U \bar{a}$ ). Notons que, puisque $S_{n}$ est un langage linéaire et que l'opération ainsi définie est une substitution rationnelle, $S_{n}^{\prime \prime}$ est également linéaire. Il en va alors de même du langage $S_{n}^{\prime}=R_{n} \cdot S_{n}^{\prime \prime}$.

Considérant alors deux nouveaux symboles \# et $\alpha$, on peut définir le langage 


$$
\hat{S}_{n}^{\prime}=\left\{\# u_{1} \alpha v_{1} \alpha w_{1} \# \cdots \# u_{p} \alpha v_{p} \alpha w_{p} \mid p \geqslant 1, u_{i}, w_{i} \in\left(z_{n} U\{\alpha\}\right)^{*} \text { et } v_{1} \ldots v_{p} \in S_{n}^{\prime}\right\}
$$

Il est facile de voir que $\hat{S}_{n}^{\prime}$ est un langage linéaire(comme $S_{n}^{\prime}$ ) et donc que le langage $\hat{S}_{n}$ défini ci-dessous l'est aussi:

$\hat{S}_{n}=\left\{g \mid g=\# \alpha a_{1} \alpha \# f \epsilon \hat{S}_{n}^{\prime}\right.$ et $\left.\# f \in K_{n}\right\}$ où

$\mathrm{K}_{\mathrm{n}}$ est le langage rationnel $\left(\{\# \alpha, \alpha\} \overline{\mathrm{Y}}\left(\mathrm{Y}^{2} \cup \mathrm{Y} \cup\{1\}\right)\right)^{*}$.

L'intérêt des langages $\hat{S}_{n}$ vient du

Lemme 1: Le langage I sur l'alphabet $X$ est linéaire propre si et seulement si il existe un entier $n$ et un homomorphisme $\phi$ de $x^{*}$ dans $z_{n}^{*}$ tel que

$$
I=\phi^{-1} \hat{S}_{\mathrm{n}} \text {. }
$$

Ce lemme est établi en étudiant d'assez près la forme normale de Greibach [2] des grammaires linéaires (la preuve est ensuite analogue à celle de [4] pour les langages algébriques). Cette étude des règles linéaires unilatères droites (i.e. de la forme $\mathrm{v} \rightarrow \mathrm{v}_{\mathrm{m}}^{\prime}$ où $\mathrm{v}^{\prime}$ est un non-terminal) suggère un traitement simultané de règles linéaires unilatères

et permet de démontrer qu'à tout langage linéaire $L$ on peut associer un langage linéaire pur L' (i.e. engendré par une gramaire linéaire sans règle unilatère) et une substitution rationnelle propre $\sigma$ telle que $L=\sigma\left(I^{\prime}\right)$. Il en résulte que le langage symétrique $S_{2}$ est un générateur fidèle [1] du cône rationnel Lin et, en vertu d'un résultat de [1] la

Proposition: Si I est un générateur du cône rationnel Iin, LU $\{1\}$ en est un générateur fidèle.

\section{B- Le résultat principal:}

Nous commençons par une remarque d'ordre général: le langage L' sur l'alphabet $X_{L}$, est élément du cylindre engendré par le langage $I_{1}$ sur l'alphabet $X_{L}$ si et seulement si il existe un langage rationnel $K$ (sur $I^{\prime}$ 'alphabet de $L^{\prime}$ ) et un homomorphisme $\phi$ du monoide libre $X_{L^{*}}^{*}$ dans $X_{L}^{*}$ tels que $L^{\prime}=\phi^{-1} L \cap K$. Ceci provient de ce que si $I^{\prime}=\phi^{-1}(I \cap R)$, on $a L^{\prime}=\phi^{-1} L \cap \phi^{-1} R$ et du fait que la composition de deux homomorphismes est un homomorphisme.

Nous considérons alors sur l'alphabet $x_{p}=\left\{x_{1}, x_{2}, \ldots, x_{p}\right\}$ les langages suivants:

- Ies rationnels $R=x_{1}^{+} x_{2}^{+} \ldots x_{p}^{+}$et $\bar{R}=x_{p}^{*^{p}}-R$.

- les langages linéaires (où $\mathrm{z}$ et $\mathrm{t}$ sont deux nouveaux symboles)

$$
\begin{aligned}
& A_{p}=\left\{z^{n_{x} x_{1}} x_{2}^{n_{2}} \ldots x_{p}^{n_{p}}+x_{p}^{n_{p}} \ldots x_{2}^{n_{2}} x_{1}^{n_{1}} z^{n} \mid n \geqslant 1, n_{i} \geqslant 1 \text { pour } i=1, \ldots, p\right\} \\
& A_{p}^{\prime}=\left\{z^{n} x_{1}^{n_{1}} x_{2}^{n_{2}} \ldots x_{p}^{n_{p}}+x_{p}^{n_{p}} \ldots x_{2}^{n_{2}} x_{1}^{n_{1}} z^{m} \mid n, m \geqslant 1, n \neq m, n_{i} \geqslant 1 \text { pour } i=1, \ldots, p\right\} \\
& I_{p}=A_{p} \cdot R \\
& L_{p}^{\prime}=A_{p}^{t} \cdot \bar{R} \\
& L_{p}^{\prime \prime}=L_{p} \cup L_{p}^{\prime} \text {. }
\end{aligned}
$$

Supposant alors que le langage $I_{p}^{\prime \prime}$ est élément du cylindre engendré par le langage $S$, et donc que $I_{p}^{\prime \prime}=\phi^{-1} S \cap \bar{K}$ où $\bar{K}$ est un langage rationnel, on établit a'abora 
qu'il existe un entier $r$ tel que

$$
\vec{K} \supseteq\left(z^{r}\right)^{+}\left(x_{1}^{r}\right)^{+} \ldots\left(x_{p}^{r}\right)^{+} t\left(x_{p}^{r}\right)^{+} \ldots\left(x_{1}^{r}\right)^{+} x_{p}^{*}
$$

(Cette preuve est identique à celle classique quí permet d'établir que si un langage rationnel $K$ contient tous les nots de la forme $a^{n} b^{m}$ où $n \neq m$, il existe un entier $r$ tel que $K$ contienne $\left(a^{r}\right)^{+}\left(b^{r}\right)^{+}$.).

Il en résulte alors facilement que si $I_{p}^{\prime \prime}=\phi^{-1} \operatorname{Sn} \bar{K}$, il existe un homomorphisme $\lambda$ tel que $L_{p}=\lambda^{-1} S \cap K$ où $K$ est le langage rationneI $K=z^{+} x_{1}^{+} x_{2}^{+} \ldots x_{p}^{+} t x_{p}^{+} \ldots x_{2}^{+} x_{1}^{+} z^{+} x_{p}^{*}$. Le leme essentiel à la preuve de notre théonie est alors le suivant:

Leme 2: Pour $p>n^{2}$, il $n^{\prime}$ existe pas a'horcomorphisme $\lambda$ tel que $I_{\mathrm{p}}=\lambda^{-1} \hat{S}_{\mathrm{n}} \cap \mathrm{K}$ qù $\mathrm{K}$ est le langage rationnel donné ci-dessus.

I'idée de la preuve de ce lemme est la suivante. Supposant que $I_{p}=\lambda^{-1} \hat{\mathrm{s}}_{n} \cap \mathrm{K}$, on s'assure que, pour tout $i=1, \ldots, p \quad \lambda x_{i}$ contient au moins une occurrence de \#. Puis on considère comment la contrainte d'égalité des nombres de $z$ à gauche de $t$ et à aroite de $t$ peut apparấtre par $\lambda$ : ceci impose que dans les mots ae $\hat{\mathrm{S}}_{\mathrm{n}}$ dont proviennent les mots de $L_{p}$ les facteurs itérants situes à droite de la dernière occurrence de $z$ (i.e. les occurrences de $x_{i}$ ) proviennent de facteurs itérants de mots de $\hat{s}_{n}$, et done d'éléments de la forme $\vec{a}_{i} a_{j}$. On trouve alors que $s i p>n^{2}$, on pourra échanger deux tels facteurs itérants ce qui. est impossible dans $I_{D}$.

La preuve du théorème est alors immédizte: supposons que Lin soit un cylindre admettant le langage $H$ comme générateur principal. Alors, puisque $H \in \operatorname{Lin}$, il existe en vertu du Iemme 1 un entier $n$ et un homomorphisme $\phi$ tel que $\mathrm{H}=\phi^{-1} \hat{\mathrm{S}}_{\mathrm{n}}$ et donc, il existe un entier $\mathrm{n}_{0}$ tel que $\hat{\mathrm{S}}_{\mathrm{n}_{0}}$ soit générateur principal du cylindre Lin. Mais alors $L_{p}^{\prime \prime}$ est, pour tout $p$, élément du cylindre engendré par $\hat{S}_{n_{0}}$; il résulte done de nos remarques préliminaires que pour tout $p$, il existe un homomorphisme $\lambda$ tel que $I_{p}=\lambda^{-1} \hat{S}_{n_{0}} \cap K$, ce qui contredit le lemme 2 .

Pour conclure, notons qu'un tel résultat négatif n'établit pas qu'il n'y ait aucun langage linéaire de complexité "maximale". En effet, bon nombre d'opérations ne modifiant pas la classe de complexité ne sont pas prises en compte ici (par exemple, 1'intersection par des langages linéaires déterministes). En outre, la preuve du résultat suggère deux possibilités : elle fournit un système générateur du cylinare Lin, la famille des $\widehat{S}_{n}$ et $i l$ semble donc que si l'on sait reconnaitre tous ces langages "raisonnablement", il en ira de même de tout langage linéaire. En outre, il apparât qu'à défeut d'un générateur du cylindre, la substitution du langage symétrique $\mathrm{s}_{2}$ dans $\hat{\mathrm{s}}_{2}$ fournit un langage qui doit contenir dans le cylindre qu'il engendre toute la famille lin (sans qu'il soit lui-même linéaire). Ainsi, la complexité des langages linéaires ne serait-elle pas plus "grande" que celle de ce dernier langage. 
[1] BOASSON L. et M. NIVAT: Sur Diverses Familles de Langages Fermées par Transductions Rationnelles.

Acta Informatica, 2, 180-188 (1973).

[2] GINSBURG S.: The Mathematical Theory of Context-Free Languages.

MC Graw HiIl (1966).

[3] GREIBACH S.: Jump paa's, Deterministic Context-Free Languages, Principal AFDL's and Polynomial Time Recognition.

Proc. of $5^{\text {th }}$ Annual ACM Symposium on Theory of

Computing, Austin, (Texas), 20-28 (1973).

[4] GREIBACH S.: The Hardest context-Free Language.

Siam J. of Comput., 2 $, n^{\circ} 4,304-330$ (1975).

I. BOASSON

U.E.R. de Mathématiques

Université de Picardie

Ad. Postale: 5, Allée Georges Rouault 75020 - PARIS (F).
M. NIVAT

U.E.R. de Mathématiques

Université Paris 7

Ad. Postale: 8 rue Portalis

75009 - PARIS (F). 